\title{
Definição de Critérios Pedagógicos e Técnicos de um Recurso Digital para Validação de Planos de Aula
}

\author{
Amanda Maria D. de Oliveira, Clésia Jordania N. da Costa, Ivanilka L. de \\ Azevedo, Vitor Henrique C. Bezerra, Dennys L. Maia \\ Instituto Metrópole Digital - Universidade Federal do Rio Grande do Norte (UFRN) \\ Lagoa Nova - 59.064-741 - Natal - RN - Brasil \\ \{amandamariadomingos, clesiaj7, vitorhenrique908\}@gmail.com, \\ \{dennys, ivanilka.azevedo\}@imd.ufrn.br
}

\begin{abstract}
This document presents the path followed by two working groups (Development and Pedagogical), in the sense of outlining a systematic, theoretical-pedagogical reference to implement a validation system for class plans in the OBAMA platform, with the goal of disseminating among teachers good pedagogical practices.
\end{abstract}

Resumo. Este artigo apresenta o percurso traçado por dois grupos de trabalho ( $G T$ de Desenvolvimento e o GT Pedagógico), no sentido de elencar um referencial teórico-pedagógico e sistemático para implementar um sistema de validação de planos de aulas na plataforma OBAMA, com o objetivo de difundir entre professores boas práticas pedagógicas.

\section{Introdução}

A busca por sistemas computacionais para a educação mais completos provoca esforços no sentido de melhorar e oferecer ao professor ferramentas que agilizem e facilitem o preparo de suas aulas, com garantia da coerência estrutural e propósitos pedagógicos que um plano de aula (PA) ou sequência didática (SD) devem conter. De acordo com dados do Centro Regional de Estudos para Desenvolvimento da Sociedade da Informação (Cetic.br), 75\% dos professores brasileiros admitem usar Internet para preparação de atividades didáticas com seus alunos. Dentre as ações mais realizadas estão a busca de exemplos de planos de aula (82\%), seguida pelo compartilhamento de conteúdos educacionais com outros professores (78\%) [Cetic.br 2017].

Nesse sentido, plataformas on-line são desenvolvidas com vistas à colaborar com a prática docente. O Portal do Professor, por exemplo, trata-se de um ambiente desenvolvido pelo Ministério da Educação (MEC) em meados dos anos 2000 que possui um documento no qual encontram-se: "dicas para a elaboração de planos de aula", elaborado pela Secretaria de Educação Básica do MEC. Esse documento socializa ao público orientações que vão desde a observação da estrutura curricular, como possibilidade daquela aula ser aproveitada em mais de um nível de ensino, à explicação do que vem a ser uma avaliação. Tais indicações oportunizam ao professor rememorar aspectos de sua formação, além de refletir sobre suas concepções pedagógicas.

Especificamente para o ensino da Matemática, a Plataforma OBAMA intenciona 
auxiliar na prática de docentes da Educação Básica com a disponibilização de mais de 500 objetos de aprendizagem (OA), que exploram conceitos matemáticos, classificados conforme critérios técnicos e pedagógicos, e uma ferramenta para elaboração colaborativa e compartilhamento online de planos de aulas [Batista et al 2017]. Os primeiros PA produzidos na referida plataforma foram elaborados, em pouco mais de um ano de seu funcionamento, em formações oferecidas a mais de 300 professores e nas pesquisas de mestrado desenvolvidas por membros da equipe. Como uma das etapas de um dos atuais projetos de pesquisa é validar esses PA elaborados, em breve, eles estarão disponíveis na OBAMA que, portanto, servirá como catalisadora de propostas de práticas inovadoras em Matemática acessíveis na internet para que professores utilizem como auxiliar em suas práticas.

Entretanto, as primeiras análises dos PA e SD produzidos demonstram que os professores têm dificuldades para desenvolver essa importante atividade docente, tanto pelo aspecto tecnológico, quanto pedagógico e do conteúdo [Silva et al 2018]. Em razão desta realidade, e da compreensão que essa plataforma pode servir como ambiente de formação docente, demandou-se ajustes na funcionalidade de elaboração de PA para que os professores tivessem suporte durante a sistematização de suas práticas de ensino em Matemática.

A reflexão, inerente à elaboração dos $\mathrm{PA}$ ou $\mathrm{SD}$, compreende todo o processo de sistematização do conhecimento desde a etapa da concepção, desenvolvimento e aplicação da proposta de ensino até o momento da avaliação, instrumento que dará suporte ao feedback dos processos de ensino e de aprendizagem. No sentido de dar suporte ao professor no processo de elaboração de PA e SD, este trabalho apresenta a narrativa da implementação de um sistema de validação para planos de aula elaborados na Plataforma OBAMA.

O presente trabalho se organiza em cinco seções que buscam trazer todo o processo de concepção do processo de validação do PA, organizado em: introdução, referencial teórico, metodologia, resultados e discussões, considerações finais e uma seção com os anexos deste artigo.

\section{Referencial Teórico}

Concebemos que os $\mathrm{PA}$ e as SD são instrumentos inerentes e essenciais à prática docente, pois ao planejar sua aula o professor tem a oportunidade de organizar suas ações de ensino em favor da aprendizagem discente e, inclusive, refletir sobre sua prática. Esse conjunto de atitudes confere o caráter pedagógico do fazer docente, atribuindo a sua práxis uma "ação consciente, intencional e planejada no processo da formação humana", conforme reitera Libâneo (1998, p.24). O PA trata-se, desse modo, em uma ferramenta didáticopedagógica pois, se bem elaborado, se constitui em um documento que sistematiza o trabalho pedagógico do professor no dia-a-dia de sala de aula, aspecto ressaltado por autores como: Oliveira (2011); Castro, Tucunduva e Arns (2008). Ademais, para autores como Vasconcellos (2000), Libâneo (1998) e Mildner (1999) a reflexão sobre a prática através do ato de planejar otimiza a intervenção sobre a realidade, possibilitando assim, uma mudança na cultura pedagógica.

Na Plataforma OBAMA, a elaboração de um PA é tratada, também, como um processo formativo para o docente que planeja utilizando os recursos da plataforma. Para fins didáticos, esse processo é dividido em três etapas visando nortear o professor no curso da elaboração do planejamento de suas aulas, visto que algumas vezes os 
professores ainda têm dificuldade, por exemplo, em definir os objetivos do seu planejamento com a finalidade de aprendizagem e não de ensino [Silva et al 2018]. Para tanto, os rótulos dos campos a serem preenchidos no formulário de criação de plano de aula são apresentados como questões a serem respondidas, como em um diálogo entre o usuário e o sistema, “(...) com isso, espera-se que o usuário compreenda o que são as informações necessárias para cadastrar um Plano de Aula e sinta-se estimulado em concluir a atividade" [Oliveira et al 2018, p. 399]. Mais que a conclusão da tarefa de planejar, é esperado que esse diálogo para criação do PA faça com que o professor reflita sobre sua proposta de aula desde o ínicio, quando é questionado sobre a instituição de ensino a qual é destinada sua proposta de aula. Essa primeira pergunta apresenta a dimensão do contexto para o qual aquele planejamento se destina, permitindo verificar se a proposta de aula está alinhada ao público-alvo. Na última etapa, na qual é possível adicionar ou não Objetos de Aprendizagem (OA) ao seu PA, o professor é colocado a analisar e justificar a integração de tecnologias, sejam digitais ou analógicas, em sua prática pedagógica [Oliveira et al, 2018].

Diante disso, sentimos a necessidade de aliar a concepção de uma boa referência de PA ou SD à uma interface para o usuário responsável pela validação a partir de um recurso digital integrado à plataforma, se fazendo necessário, para tanto, iniciar com 0 levantamento de requisitos. Para Bezerra (2007, p.23), é nesse momento que “(...) a equipe de desenvolvimento tenta entender o domínio que deve ser automatizado pelo sistema de software". Nesse contexto, de acordo com Bezerra (2007, p.23), o domínio pode ser definido como "(...) a área de conhecimento ou de atividade específica caracterizada por um conjunto de conceitos e de terminologia compreendidos por especialista nessa área".

O autor explica que o levantamento de requisitos é, também, um momento de estudo das necessidades dos usuários interessados por parte dos desenvolvedores, que antecede e justifica a necessidade de implementação do recurso [Bezerra 2007]. Por isso é preciso que esses estudos sejam documentados de forma que, em momentos futuros, outros desenvolvedores possam entender as motivações para o desenvolvimento de tal recurso. Essa documentação pode ser feita utilizando diagramas, descrições textuais ou mesmo esboços e registros de conversas entre equipe de desenvolvimento e usuários (gravações de áudio, vídeo ou atas de reuniões, por exemplo).

Na seção seguinte, apresentamos como se deu o percurso metodológico adotado no desenvolvimento deste trabalho que culminou na sistematização do ambiente de validação de PA e SD da Plataforma OBAMA.

\section{Metodologia}

Este trabalho está dividido em duas partes essenciais para o desenvolvimento de um recurso digital cuja finalidade é pedagógica: i) a concepção e definição dos aspectos pedagógicos; e ii) a implementação do recursos digital em si. Por isso, foram utilizadas metodologias distintas, embora integradas, para cada uma das etapas.

\subsection{Definição dos critérios pedagógicos}

Definido o processo de criação de PA dentro da Plataforma OBAMA, foi então organizada, na equipe de colaboradores da Plataforma, um Grupo de Trabalho (GT) Pedagógico para elaborar critérios de avaliação de cada ponto do PA. Esse grupo, portanto, toma como base as teorias e trabalhos que apresentam o PA como uma 
ferramenta de avaliação do aluno e de autoavaliação do professor, que toma esse esquema como o roteiro de sua prática profissional. Nesse sentido, foi realizada uma revisão bibliográfica que ocorreu de dezembro de 2018 a março de 2019, com vistas a subsidiar a base teórica apresentada e assim garantir a constituição de um sistema de validação de PA produzidos em uma plataforma virtual. Para cada ponto do PA, é feita uma análise do que foi proposto e se atende ou não a especificidade de cada item. Esse e outros pontos serão analisados com um olhar que busca enxergar a coerência entre a intenção do que se quer ensinar e as estratégias, procedimentos e intervenções utilizadas para tal, mediante o processo de avaliação adotada.

Como a avaliação desses planos tem por finalidade publicizar boas práticas pedagógicas, proporcionando aos professores orientações sobre a elaboração de planos de aula, a exemplo da Plataforma MEC de Recursos Educacionais Digitais (MEC RED)1 das demais plataformas educacionais, nos dispomos a usar uma metodologia de validação que busca analisar cada um dos itens informados no PA ou SD elaborados pelo professor, na busca da conexão desses pontos com o objetivo pedagógico daquele plano. Ao final, foram listados oito critérios pedagógicos que congregam para propostas de boas práticas para aulas em Educação Matemática.

A concepção do sistema de validação tem como marco a definição dos critérios usados para avaliar cada tópico dos PA submetidos na Plataforma OBAMA, discutido pelo GT Pedagógico. Seguindo com a implementação do recurso digital para validação, que foi iniciado com a elaboração do fluxo de navegação, e então realizada a prototipagem das telas para poder ser implementado e testado. A elaboração de um diagrama de fluxo de navegação auxiliou ao Grupo de Trabalho de Desenvolvimento (GT Devs) a entender as demandas apontadas pelo GT Pedagógico.

\subsection{Definição e implementação de critérios técnicos}

O processo de desenvolvimento do recurso de avaliação de planos de aula foi iniciado com a análise das demandas pedagógicas levantadas. Para tanto, foi elaborado um diagrama para representação do de fluxo de navegação (anexo: figura 1), o qual representa os passos a serem dados pelos membros da equipe de avaliação dentro da Plataforma OBAMA para a realização da tarefa de validar um PA. A definição do fluxo foi feita a partir da elaboração de um diagrama que apresenta os objetivos a serem alcançados para a validação do PA e quem está realizando tal objetivo. O diagrama (anexo: figura 1) foi elaborado em três etapas: $i$ ) definição dos objetivos a serem alcançados, em sequência, para a revisão do PA; ii) Atribuição aos devidos atores (usuários e sistema) seus objetivos; iii) Mapeamento dos fluxos alternativos (situações em que há desvio do fluxo ideal no processo de validação).

Após análise das demandas, foi então realizada a prototipagem das telas do recurso de validação de PA. Com a validação dos protótipos de tela apresentados pelo GT Devs para o GT Pedagógico, foi então implementado o recurso de validação. Todo o processo de elaboração de diagramas e prototipagem das telas foi realizado com o editor de diagramas Draw.io integrado ao Google Drive, de forma a possibilitar o trabalho colaborativo entre os membros do GT Devs. A prototipagem de telas representa os

1 Criada em 2015 por meio de uma iniciativa do MEC, que traz dicas gerais para elaboração de planos de aula estruturados e, também, disponibiliza recursos dos principais portais educativos do Brasil, além de divulgar os planos de aulas de todas as disciplinas. 
aspectos visuais (telas) e de interação que auxiliarão no alcance dos objetivos definidos a partir da análise das demandas do GT Pedagógico.

O recurso de validação de PA foi implementado nas linguagens de programação JavaScript, CSS, HTML e Java com o framework Model View Controller VRaptor. Essas mesmas linguagens e framework são utilizados para o desenvolvimento dos demais recursos da Plataforma OBAMA, sendo as três primeiras necessárias para a criação de páginas dinâmicas em sistemas e sites da web e a última utilizada para a implementação das regras de funcionamento da plataforma e comunicação com o banco de dados.

A seguir são apresentados os critérios definidos para validação de um PA na Plataforma OBAMA e o recurso digital desenvolvido para a realização de tal tarefa.

\section{Resultados e discussões}

Diante do exposto, o GT Pedagógico considerou que para o PA ou SD ser considerado como boa prática, e desse modo publicizado na plataforma, necessitaria atender a sete critérios considerados relevantes para sua coerência, são eles: $i$ ) público-alvo; ii) tempo adequado ao desenvolvimento da atividade; iii) descritor(es) ou habilidades em Matemática; iv) objetivo geral claro e focado no aluno; $v$ ) objetivos específicos claros e relacionado a todas as etapas implícitas para o alcance do objetivo geral; vi) metodologia conectada aos objetivos propostos; e vii) avaliação coerente com os objetivos. Definidos os critérios para validação de PA na Plataforma OBAMA pelo GT Pedagógico, ao GT Devs coube a responsabilidade de implementar o recurso digital que daria suporte a esta tarefa. O início da implementação de um software ou recurso para um software já existente se dá com o estudo do que será implementado. Para isso, o GT Devs optou por delinear o fluxo da navegação do usuário dentro sistema durante a validação de um PA.

Cada objetivo é apresentado no diagrama como um retângulo que contém uma sentença indicando o objetivo a ser alcançado. Todos os retângulos possuem ligações com outros retângulos através de setas, cujas pontas indicam a tarefa precedente e a próxima tarefa a ser realizada. Cada retângulo é indicado por uma cor que designa quem é o ator responsável por aquele objetivo dentro do fluxo. É válida a regra da hierarquia entre os perfis de usuários, que será melhor detalhada posteriormente, nesse caso o que é o indicado como uma tarefa do usuário autor, por exemplo, pode ser realizada também por qualquer um dos outros perfis de usuário.

Inicialmente era previsto que um PA poderia ser submetido para validação por qualquer usuário cadastrado na plataforma. Após submetido, este PA estaria automaticamente disponível e qualquer um dos usuários com perfil de acesso do tipo Revisor poderia iniciar a validação. Porém, se feito dessa maneira, o processo de validação não preveniria situações em que dois revisores iniciem a revisão de um mesmo PA, ou mesmo que a revisão seja realizada pelo próprio autor do PA, caso esse tenha perfil de Revisor. Com o estudo do fluxo definido, foi percebido que há mais que dois usuários (autor e revisor) envolvidos na tarefa de validação de um PA.

Como mostra o diagrama de caso de uso das principais funcionalidades da Plataforma OBAMA (anexo: figura 2), os perfis dos usuários da plataforma respeitam a uma hierarquia, cuja menor atribuição de funcionalidades de um usuário logado é do usuário com perfil do tipo Padrão (Usuário). Acima deste está o Revisor que tem as mesmas atribuições do usuário Padrão com o adicional de acesso à funcionalidade de revisão de $\mathrm{PA}$ e habilitar e desabilitar OA. Por fim, está o usuário com perfil de 
Administrador que é capaz de, além de tudo que é atribuído ao Revisor, habilitar e desabilitar um usuário e alterar o perfil dos demais usuários.

Quando confrontados, o diagrama de caso de uso inicialmente proposto e o diagrama de fluxo de navegação para validação de um PA, fez-se necessário definir outros dois perfis de usuário que dariam conta de cobrir as lacunas deixadas pelo planejamento inicial do caso de uso de validação de PA. Esses novos perfis são: Revisor-Pesquisador e Revisor-Sênior.

Ao Revisor-Pesquisador é dada a possibilidade de revisar seus PA e de outros autores. Esse perfil se fez necessário na medida em que a equipe de revisão é, também, a equipe que organiza e faz a mediação nas formações sobre o uso da plataforma, sendo portanto, também, coautores dos PA elaborados durante as formações. Neste caso, é atribuído o título de Pesquisador a este perfil, dada sua especificidade no trabalho de formar professores que desenvolvem boas práticas pedagógicas utilizando a Plataforma OBAMA. Enquanto isso, ao Revisor-Sênior é atribuída a demanda de delegar um Revisor-Pesquisador para validar o PA e finalizar o processo de validação, verificando se a validação do Revisor-Pesquisador foi coerente ao proposto, no tocante aos aspectos pedagógicos definidos. O Revisor-Sênior veio, também, para que a validação do PA se desse em dois passos, reforçando a qualidade no processo de validação deste documento. Para tanto, esse papel será atribuído apenas aos membros do GT Pedagógico que detenham título de pós-graduação stricto sensu, prioritariamente, em nível de Doutorado.

Após definido o fluxo de navegação para realização da validação de um PA, o diagrama foi apresentado ao GT Pedagógico que aprovou a proposta. Foi então realizada a prototipagem das telas que são apresentadas aos usuários durante a validação do PA. A primeira tela envolvida no processo de validação é a tela de cadastro de PA, dado que o processo de validação é iniciado na escrita do PA quando o professor está respondendo as perguntas que compõem o formulário de cadastro de PA. Neste caso, não foram necessárias mudanças no formulário de cadastro em si. No momento do envio do PA para validação passou a ser exibida uma tela que apresenta o "Termo de consentimento com o processo de validação e publicação de Planos de Aula da Plataforma OBAMA", como mostra a figura 3 (anexo).

Apresentar os termos e condições para validação e publicação de PA na Plataforma OBAMA se faz necessário por dois motivos, primeiro porque todas a publicações (de OA e PA) por meio da Plataforma terão a mesma licença livre atribuída à plataforma (CC-BY-SA), ou seja, estarão sob uma licença criativa que garante o direito de referência ao autor e suas réplicas e cópias devem se manter sob a mesma licença. $\mathrm{O}$ segundo motivo é que a validação, enquanto processo avaliativo da proposta pedagógica, se constitui, também, etapa do processo formativo desse profissional, portanto, devem estar explícitos quais aspectos serão considerados durante a validação.

Além disso, foi preciso projetar uma tela que ainda não existia na plataforma, a de validação de PA. Neste caso, foram levadas em consideração as experiências dos membros do GT Pedagógico para definição do layout desta tela. Dado que todos os participantes do referido GT fazem uso constante de formulários digitais do Google Formulários, o primeiro protótipo foi construído pelo próprio GT Pedagógico utilizando esta ferramenta. Esse passo se fez necessário para que os próprios usuários da funcionalidade e proponentes dessa demanda entendessem quais tipos de campos de entrada de informação seriam necessários no formulário. 
Como os membros do GT Pedagógico já estavam elaborando os critérios para validação do PA, eles foram considerados os mais aptos para definir como esses critérios seriam verificados. Com isso, foi definido que cada critério seria considerado como uma questão objetiva de múltipla escolha e haveria, também, um campo de texto para a escrita das considerações finais do revisor (anexo: figura 4). No topo da tela há um link que permite ao revisor visualizar ou baixar um arquivo PDF com o conteúdo do PA. Por fim, no rodapé do formulário, o revisor tem dois botões que representam sua decisão em relação ao próximo passo na validação do PA: Validar e Enviar para correção.

O GT Pedagógico descartou a hipótese de rejeição de um PA como veredito final da validação, dada a intenção formativa do processo de validação de PA, cujo objetivo não é elencar boas práticas pedagógicas, mas incentivar a construção de PA que apresentem tal prática. Desta forma, após enviado para validação, o PA só sairá do fluxo de validação quando for publicizado. Para tanto, quando necessário ajustes, todos os usuários-autores do PA serão notificados por e-mail com uma mensagem informando o parecer do revisor e os critérios que o PA atendeu ou não. Todo o processo de validação será em formato de blind review, ou seja, o revisor não saberá quem são os autores. Aplicou-se uma exceção aos casos já informados aqui, como o do Revisor-Pesquisador.

A validação final do Revisor-Sênior é realizada em uma tela semelhante à de validação de PA. Neste caso, se diferencia apenas por apresentar as informações já preenchidas e o botão Validar é substituído por Publicar. Caso o PA seja retornado aos autores para correção, essa correção se dará na tela de edição do PA. Dada a semelhança que as telas para estes dois objetivos têm com as telas para edição e validação de PA, seus protótipos seguem o mesmo padrão apresentado nas figuras 3 e 4 , respectivamente.

Após aprovação dos protótipos de telas, as atividades de desenvolvimento foram prosseguidas com a implementação do sistema de validação. No momento da elaboração deste artigo, a implementação estava em estágio de testes pelos membros do GT Devs, responsáveis pela implementação, para então ser avaliada pelo GT Pedagógico e por fim disponibilizada para os demais usuários da Plataforma OBAMA.

\section{Considerações finais}

Com a implementação e uso efetivo da funcionalidade de elaboração de PA de forma colaborativa e posteriormente sua publicação na plataforma, mediante avaliação, esperamos contribuir com a ampliação de boas práticas de ensino de Matemática ao propor a reflexão por ocasião da sistematização dos PA e SD. Ressaltamos, com isso, atenção aos critérios adotados, para promoção da reflexão e aperfeiçoamento das práticas de ensino da Matemática mediante o feedback do material submetido à Plataforma OBAMA e ainda ao nosso objetivo em difundir e reconhecer publicamente em ambiente virtual tais referências de boas práticas de ensino.

Durante o desenvolvimento do recurso aqui apresentado, toda a equipe envolvida pôde consolidar a compreensão da importância de uma equipe multidisciplinar para a concepção e implementação de um recurso digital com fins pedagógicos. Bem como, para o GT Devs, a melhor compreensão e implementação do recurso de validação de PA foi viabilizada a partir de documentos e registros realizados pelos idealizadores das versões iniciais de tal recurso, por meio de diagramas, protótipos e artigos científicos. Enquanto para o GT pedagógico, enumerar os critérios de validação e conceber um sistema de validação consistente na idealização de um PA coerente nos aspectos pedagógicos, só foi 
instituído a partir da busca e estudo do referencial teórico e da discussão entre os colaboradores, o que garantiu a versão atual do sistema.

\section{Referências}

Batista, S.; Brito, D; Melo, E; Oliveira, A.; Oliveira, N.; Maia, D. (2017). "Reconstrução de um repositório de objetos de aprendizagem para Matemática". In: Anais do II Congresso sobre Tecnologias na Educação, Mamanguape/PB.

Bezerra, E. (2007). "Princípios da Análise e Projetos de Sistemas com UML". Elsevier Editora Ltda. São Paulo.

Castro, P.; Tucunduva, C.; Arns, E. (2008). “A Importância Do Planejamento das Aulas Para Organização do Trabalho do Professor em sua Prática Docente". Athena, Revista Científica de Educação, v.10, n.10, Jan./Jun.

Cetic.Br. (2017). "Pesquisa sobre o uso das tecnologias de informação e comunicação nas escolas brasileiras: TIC Educação”. Brasília: Cgi.Br/Nic.Br.

Fiorentini, D.; Lorenzato, S. (2006). "Investigação Em Educação Matemática: Percursos Teóricos E Metodológicos”. Campinas, SP: Autores Associados, 2006.

Libâneo, J. (1998). "Didática”. 2 ${ }^{\text {a }}$ Ed. São Paulo: Cortez.

Mildner, T. (1999). "Avaliação da aprendizagem escolar numa visão de articulação com os processos de planejamento e de implementação de ensino-aprendizagem”. Estudos em avaliação educacional. São Paulo: Fund. Carlos Chagas, n.20, p. 95-128.

Oliveira, A.; Oliveira, A.; Batista, S.; Nascimento, I.; Azevedo, D.; Lima, R.; Oliveira, N.; Maia, D. (2018). "Processo de desenvolvimento de uma ferramenta destinada à elaboração de planos de aula de forma colaborativa". In: Anais do III Congresso sobre Tecnologias na Educação, p.392-403, Fortaleza/CE.

Oliveira, M. (2011). "Plano de Aula: ferramenta pedagógica da prática docente". Pergaminho. Patos de Minas, MG: Unipam, (2): 121-129.

Pereira, D.; Rocha, S.; Chaves, P. (2016). "O conceito de práxis e a formação docente como Ciência da Educação". Revista de Ciências Humanas, v.17, p.31-45.

Silva, A.; Silva, A.; Souza-Filho, M.; Melo, E.; Azevedo, I.; Maia, D. (2018) "Análise de planos de aula para o ensino de Matemática com uso de objetos de aprendizagem". Revista Tecnologias na Educação, v.26, p.135-155.

Vasconcellos, C. (2000). "Planejamento: projeto de ensino-aprendizagem e projeto político pedagógico". 9ed. São Paulo: Libertad.

\section{Anexos}




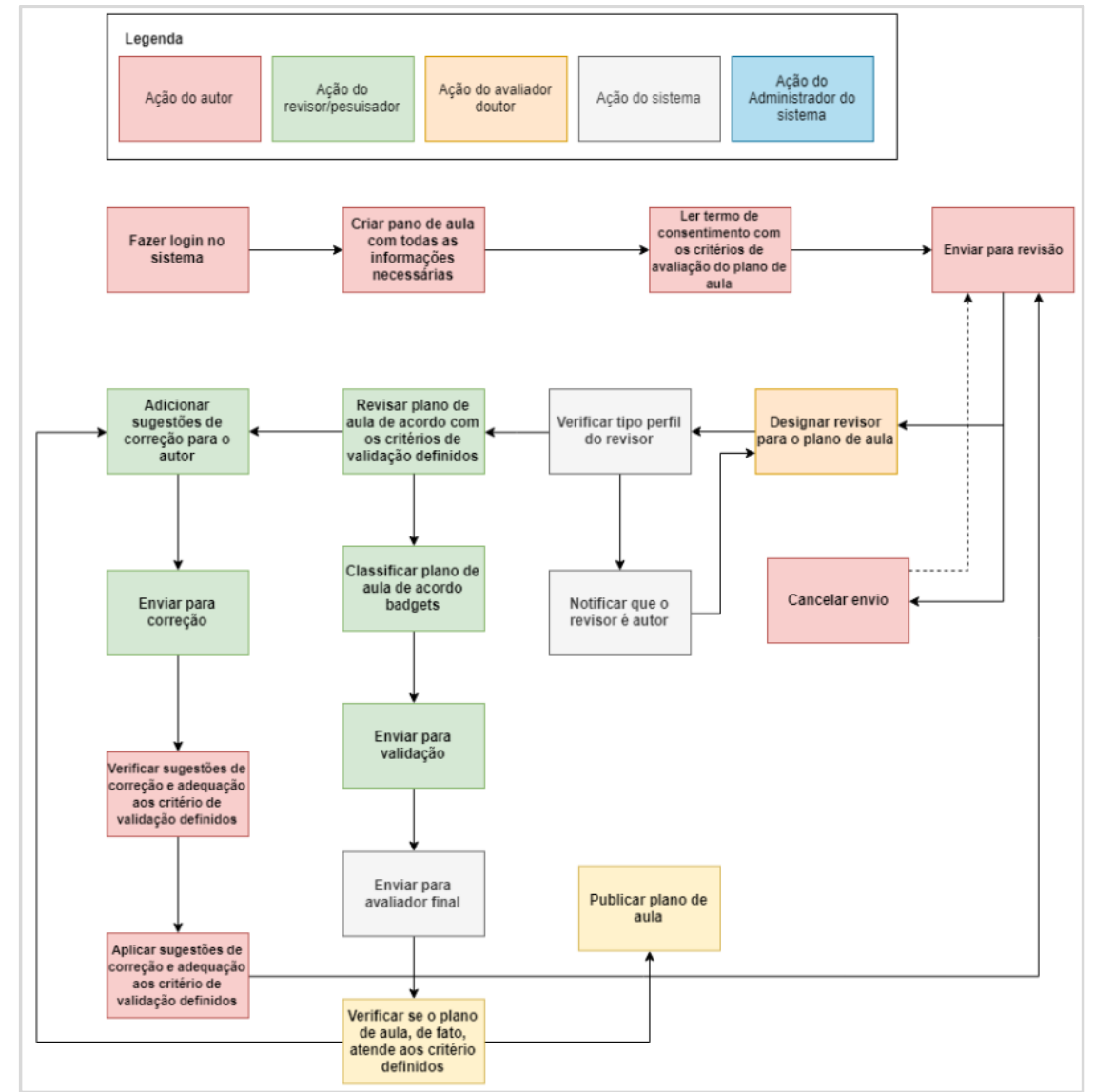

Figura 1. Diagrama de fluxo de navegação para validação de um PA na Plataforma OBAMA

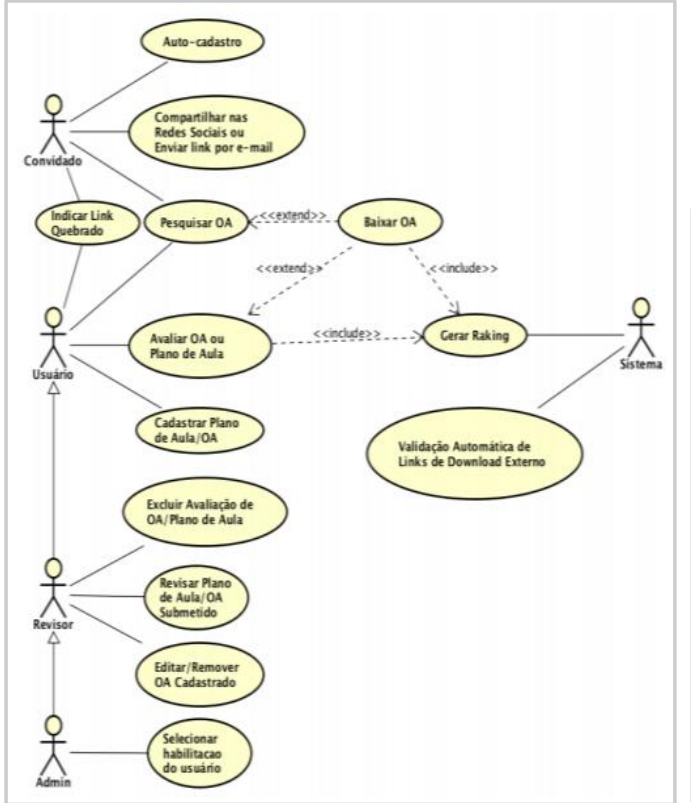

Figura 2. Diagrama de Casos de Uso da Plataforma OBAMA (Fonte: Batista et al 2017)

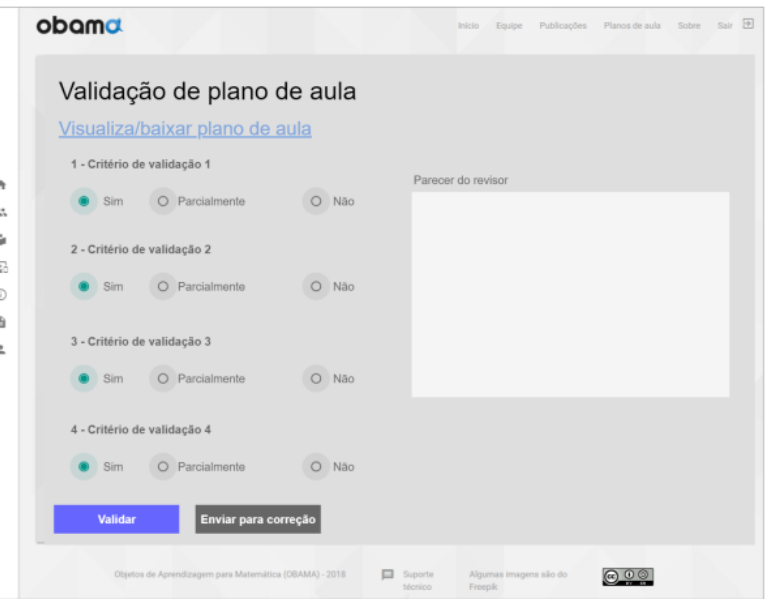

Figura 4. Protótipo de tela do formulário de validação de PA 


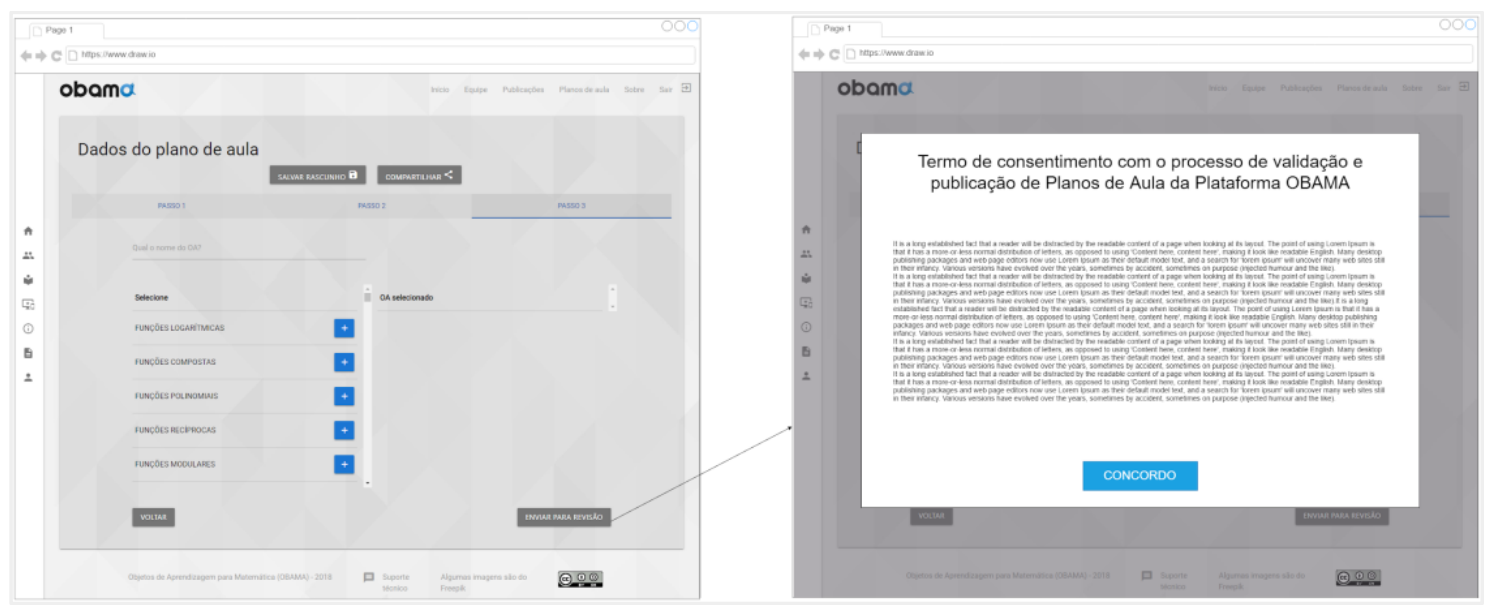

Figura 3. Finalização do cadastro de PA e Termo de consentimento com o processo de validação e publicação de Planos de Aula da Plataforma OBAMA 